\title{
DE PAEPE'S DISC HAS NONTRIVIAL POLYNOMIAL HULL
}

\author{
A. G. O'FARRELL AND M. A. SANABRIA-GARCÍA
}

AbSTRACT

The topological disc (De Paepe's)

$$
P=\left\{\left(z^{2}, \bar{z}^{2}+\bar{z}^{3}\right):|z| \leqslant 1\right\} \subset \mathbb{C}^{2}
$$

is shown here to have non-trivial polynomially convex hull. In fact, the authors show that this holds for all discs of the form $X=\left\{\left(z^{2}, f(\bar{z})\right):|z| \leqslant r\right\}$, where $f$ is holomorphic on $|z| \leqslant r$, and $f(z)=z^{2}+a_{3} z^{3}+\ldots$, with all coefficients $a_{n}$ real, and at least one $a_{2 n+1} \neq 0$.

\section{Introduction}

The polynomial hull of a compact set $X \subset \mathbb{C}^{n}$ is defined as

$$
\hat{X}=\left\{z \in \mathbb{C}^{n}:|p(z)| \leqslant \sup _{X}|p| \text { for every holomorphic polynomial } p\right\} .
$$

This object is important for approximation theory, Banach algebras and spectral theory, and has been the subject of much study. It is, however, usually a difficult problem to give an explicit description of the polynomial hull of specific compact sets $X$. It is known that the polynomial hull of a smooth arc in $\mathbb{C}^{n}$ is itself, and that the polynomial hull of a smooth simple closed curve $\Gamma$ is either itself, or is obtained by adjoining a one-dimensional analytic variety $\hat{\Gamma}$ whose boundary is $\Gamma$ (see [1, Chapter 12] and the references therein).

For smooth surfaces $X \subset \mathbb{C}^{2}$, some problems remain, but the situation is pretty well understood when $X$ is diffeomorphic to the closed unit disc, and either $X$ is totally real or $X$ has an isolated complex tangent plane that satisfies a nondegeneracy condition; see the survey [7] for an account of the local theory.

One of the problems at the frontier of our understanding concerns the nature of the hull of a topological disc in $\mathbb{C}^{2}$ which is smooth except for a single singular point. Another problem concerns the hull of a pair of smooth discs having a single common point, at which they are tangent to one another. This paper addresses a problem that relates to both cases.

In 1984, P. J. de Paepe (see [4]) asked about the hull of the topological disc

$$
P=\left\{\left(z^{2}, \bar{z}^{2}+\bar{z}^{3}\right):|z| \leqslant 1\right\} \subset \mathbb{C}^{2} .
$$

There have been a number of results about 'similar' discs in the interim, but the problem of whether or not $P$ equals its own hull has resisted attack until now. We solve this problem (in the context of a rather more general class of such problems), and we describe a related result about pairs of tangent discs. The method of proof uses ideas from complex dynamical systems applied to the composition of two

Received 26 April 2001.

2000 Mathematics Subject Classification 32E20.

Supported by a grant of the Gobierno Autónomo de Canarias. 
non-commuting involutions associated with the disc. The inspiration for the idea came from the great Acta paper [3] of Moser and Webster about normal forms for surfaces in $\mathbb{C}^{2}$ having an isolated complex tangent of (Bishop) elliptic type.

There are two holomorphic involutions of a disc naturally associated to De Paepe's problem. The problem considered by Moser and Webster gives rise to a pair of involutions of a neighbourhood of 0 in $\mathbb{C}^{4}$. Moser and Webster imbedded the composition of their involutions into a flow, and then complexified the flow to obtain orbits which were analytic discs. We follow a broadly similar strategy, although the detail of the execution is quite different.

\section{The main result}

THEOREM 2.1. Let $f(z)$ be analytic on a neighbourhood of the origin, and such that $f(z)=z^{2}+a_{3} z^{3}+\ldots$, with all coefficients $a_{n}$ real, and at least one $a_{2 n+1} \neq 0$. Then for $r>0$ small enough, the compact topological disc

$$
X=\left\{\left(z^{2}, f(\bar{z})\right):|z| \leqslant r\right\} \subset \mathbb{C}^{2}
$$

has non-trivial polynomial hull.

Proof. Consider the non-trivial holomorphic involution $\tau_{1}(z)=-z$, and let $\tau_{2}$ be the non-trivial holomorphic involution defined by $f\left(\tau_{2}(z)\right)=f(z)$ near 0 .

Let $\varphi=\tau_{1} \circ \tau_{2}$. Then

$$
\varphi=z+b_{2} z^{2}+\ldots
$$

near 0 , with real coefficients $b_{n}$. (For De Paepe's function, $b_{2}=1$. In general, $\varphi(z)=z+b_{p} z^{p}+\ldots$ for some $p \geqslant 2$, with $b_{p} \neq 0$.) We note that as $\varphi$ has real coefficients and is the composition of two involutions, either the positive real axis is an attracting direction and the negative a repelling one (near 0 ), or vice versa.

From the theory of one-dimensional complex dynamical systems (see [2, Chapter 2]), there exists a region $D$ (a 'petal') with $0 \in \operatorname{bdy} D$ and such that $\varphi: D \rightarrow D$, and the action of $\varphi$ on $D$ is conjugate to a translation $z \mapsto z+1$ on a region $D^{\prime}$ that contains a right half-plane $\Pi^{+}$. Therefore, we can find a region $D^{\prime \prime} \subset D$ and a semi-flow $\varphi_{\tau}(z)$ (for $\tau \in \mathbb{H}$ and $z \in D^{\prime \prime}$ ) on $D^{\prime \prime}$, where $\mathbb{H}$ is the right half-plane semigroup $\mathbb{H}=\{\tau \in \mathbb{C}: \mathfrak{R} e \tau \geqslant 0\}$, with $\varphi_{1}=\varphi$. Moreover, $\varphi_{s}(x) \in \mathbb{R}$ for $s \geqslant 0$ and $x \in D^{\prime \prime} \cap \mathbb{R}$, an open interval having 0 as one endpoint (see Figure 1).
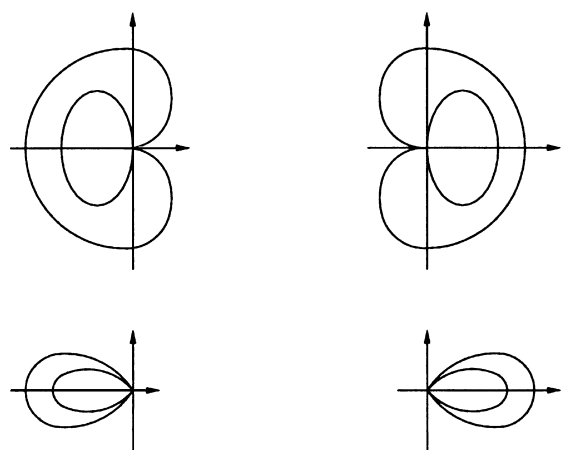

Figure 1. Possible $D$ shapes with $D^{\prime \prime}$ inside. 
Let $\zeta_{0}$ be any point on $D^{\prime \prime} \cap \mathbb{R}$. Then $\varphi_{s}\left(\zeta_{0}\right)$ is real-valued for $s \geqslant 0$, so

$$
\varphi_{\bar{\tau}}\left(\zeta_{0}\right)=\overline{\varphi_{\tau}\left(\zeta_{0}\right)}
$$

by the reflection principle, for all $\Re e \tau \geqslant 0$.

Let $\Omega$ denote the strip $\{\tau \in \mathbb{C}: 0<\Re e \tau<1 / 2\}$, and let $L=\{\tau \in \mathbb{C}: \mathfrak{R} e \tau=0\}$ and $R=\{\tau \in \mathbb{C}: \Re e \tau=1 / 2\}$ be its bounding straight lines.

Define

$$
\begin{aligned}
\Phi(\tau) & =\left(\varphi_{\tau}\left(\zeta_{0}\right),-\varphi_{1-\tau}\left(\zeta_{0}\right)\right), \\
F\left(z_{1}, z_{2}\right) & =\left(z_{1}^{2}, f\left(z_{2}\right)\right), \\
G & =F \circ \Phi .
\end{aligned}
$$

Then $G(\Omega)$ is a nonsingular one-dimensional analytic variety, imbedded in $\mathbb{C}^{2}$, with boundary $G(L) \cup G(R) \cup\{0\}$.

Fix $\tau=i t \in L$. Then, letting $z=\varphi_{i t}\left(\zeta_{0}\right)$, we obtain

$$
\Phi(\tau)=\left(\varphi_{i t}\left(\zeta_{0}\right),-\varphi\left(\varphi_{-i t}\left(\zeta_{0}\right)\right)\right)=(z,-\varphi(\bar{z})),
$$

and

$$
G(\tau)=\left(z^{2}, f(-\varphi(\bar{z}))\right)=\left(z^{2}, f(\bar{z})\right),
$$

so $G(L) \subset X$.

On the other hand, if $\tau=1 / 2+i t \in R$, then $1-\tau=1 / 2-i t=\bar{\tau}$, so letting $z=-\varphi_{\tau}\left(\zeta_{0}\right)$, we obtain

$$
\Phi(\tau)=\left(\varphi_{1 / 2+i t}\left(\zeta_{0}\right),-\varphi_{1 / 2-i t}\left(\zeta_{0}\right)\right)=(-z, \bar{z}),
$$

and

$$
G(\tau)=\left(z^{2}, f(\bar{z})\right),
$$

so $G(R) \subset X$, as well. Therefore $G($ bdy $\Omega) \subset X$.

Since $X \backslash\{0\}$ is a smooth totally real submanifold of $\mathbb{C}^{2}$, its intersection with $G(\Omega)$ is at most a one-dimensional set, so $G(\Omega) \notin X$. Also, $G(\Omega)$ belongs to the polynomial hull of $X$ (by the maximum principle), so $X$ has non-trivial polynomially convex hull.

We note that the proof of Theorem 2.1 exhibits a three-manifold in the polynomial hull of $X$, which is fibred by a one-parameter family of non-singular complex curves. We conjecture that this is the entire polynomial hull, in the case of De Paepe's disc.

\section{Concluding remarks}

It has been observed in the past (see De Paepe's survey [6]) that the hull of De Paepe's disc is related to the hull of a pair of discs, disjoint except for a single common tangent at the origin. By much the same method, we can show that the following proposition is true.

Proposition 3.1. Let $f(z)$ and $g(z)$ be analytic in a neighbourhood of the origin such that $f(0)=g(0), f^{\prime}(0)=g^{\prime}(0) \neq 0$, and $f^{\prime \prime}(0) \neq g^{\prime \prime}(0)$, and let

$$
X=\left\{(z, w) \in \mathbb{C}^{2}: w=f(\bar{z}) \text { or } w=g(\bar{z})\right\} .
$$

Suppose also that $f$ and $g$ are real-valued on the real axis (near 0$)$. Then $X$ has non-trivial polynomial hull. 
Proof. Without loss of generality, we can suppose that $f(0)=g(0)=0$. By applying the holomorphic automorphism $(z, w) \mapsto(f(z), w)$, we reduce to the case where $f(z)=z, g(z)$ is real-valued on the real axis and $g^{\prime}(0)=1$, while $g^{\prime \prime}(0) \neq 0$.

We let $\varphi=g$. The conditions above imply that either the positive real axis is an attracting direction and the negative is repelling, or vice versa.

We proceed then as in the proof of Theorem 2.1, but we now consider

$$
\Phi(\tau)=\left(\varphi_{\tau}\left(\zeta_{0}\right), \varphi_{1-\tau}\left(\zeta_{0}\right)\right) .
$$

Then $\Phi(\Omega)$ is a non-singular analytic curve in $\mathbb{C}^{2}$, with boundary $G(L) \cup G(R) \cup\{0\}$ contained in $X$. We proceed to check that this boundary lies in $X$.

Fix $\tau=i t \in L$. Then, letting $z=\varphi_{i t}\left(\zeta_{0}\right)$, we obtain

$$
\Phi(\tau)=\left(\varphi_{i t}\left(\zeta_{0}\right), \varphi\left(\varphi_{-i t}\left(\zeta_{0}\right)\right)\right)=(z, \varphi(\bar{z}))=(z, g(\bar{z}))
$$

so $\Phi(L) \subset X$.

On the other hand, if $\tau=1 / 2+i t \in R$, then $1-\tau=1 / 2-i t=\bar{\tau}$, so letting $z=\varphi_{\tau}\left(\zeta_{0}\right)$, we obtain

$$
\Phi(\tau)=\left(\varphi_{1 / 2+i t}\left(\zeta_{0}\right), \varphi_{1 / 2-i t}\left(\zeta_{0}\right)\right)=(z, \bar{z}),
$$

so $\Phi(R) \subset X$, as well. Therefore $\Phi($ bdy $\Omega) \subset X$, and the same argument as in the proof of Theorem 2.1 shows that $X$ has non-trivial polynomial hull.

Remark 3.2. De Paepe previously observed that the disc

$$
X=\left\{\left(z^{2},\left(\frac{\bar{z}}{1+\bar{z}}\right)^{2}\right):|z| \leqslant \frac{1}{2}\right\}
$$

is non-convex (see [5]). In this particular case, the semigroup action that occurs in the above proof is realized by a semigroup of linear fractional transformations. The main point of the above argument is the method by which a suitable semigroup is identified in the general case.

REMARK 3.3. Using the proper holomorphic map $\left(z_{1}, z_{2}\right) \mapsto\left(z_{1}^{2}, z_{2}\right)$, we see from Theorem 2.1 that the pair of discs

$$
\left\{\left(z, \bar{z}^{2}+\bar{z}^{3}\right)\right\} \cup\left\{\left(z, \bar{z}^{2}-\bar{z}^{3}\right)\right\}
$$

has non-trivial polynomial hull. These discs are both totally real on a deleted neighbourhood of 0 , and have second-order contact at 0 .

REMARK 3.4. We note that Proposition 3.1 is still valid under the weaker assumption of $g \circ f^{-1}$ being real-valued on the real axis. On the other hand, the same argument shows that the union of the two tangent discs

$$
X=\left\{(z, w) \in \mathbb{C}^{2}: w=\bar{z} \text { or } w=g(\bar{z})\right\}
$$

where the holomorphic function $g$ is real-valued on the real axis and $g(0)=0$, $g^{\prime}(0)=1, g^{\prime \prime}(0)=\ldots=g^{(2 n-1)}(0)=0$, and $g^{(2 n)}(0) \neq 0$, is non-polynomially convex. 


\section{References}

1. H. Alexander and J. Wermer, Several complex variables and Banach algebras, 3rd edn (Springer, New York, 1998).

2. L. Carleson and T. W. Gamelin, Complex dynamics (Springer, New York, 1993).

3. J. K. Moser and S. M. Webster, 'Normal forms for real surfaces in $\mathbb{C}^{2}$ near complex tangents and hyperbolic surface transformations', Acta Math. 150 (1983) 255-296.

4. P. J. DE Paepe, 'Approximation on disks', Proc. Amer. Math. Soc. 97 (1986) 299-302.

5. P. J. DE PAEPE, 'Approximation on a disk. I', Math. Z. 212 (1993) 145-152.

6. P. J. DE PAEPE, 'Eva Kallin's lemma on polynomial convexity', Bull. London Math. Soc. 33 (2001) 1-10.

7. A. Sanabria-García, 'Polynomial hull of smooth discs: a survey', Irish Math. Soc. Bull. 43 (2000) $135-153$.

Department of Mathematics

National University of Ireland

Maynooth

Co. Kildare

Ireland

aof@maths.may.ie

manuel@maths.may.ie 